\title{
Extrapolation of a nonlinear force-free field containing a highly twisted magnetic loop
}

\author{
G. Valori ${ }^{1}$, B. Kliem ${ }^{1}$, and R. Keppens ${ }^{2}$ \\ 1 Astrophysical Institute Potsdam, An der Sternwarte 16, 14482 Potsdam, Germany \\ e-mail: gvalori@aip.de \\ 2 FOM-Institute for Plasma Physics Rijnhuizen, PO Box 1207, 3430 BE Nieuwegein, The Netherlands
}

Received 14 September 2004 / Accepted 6 November 2004

\begin{abstract}
The stress-and-relax method for the extrapolation of nonlinear force-free coronal magnetic fields from photospheric vector magnetograms is formulated and implemented in a manner analogous to the evolutionary extrapolation method. The technique is applied to a numerically constructed force-free equilibrium that has a simple bipolar structure of the normal field component in the bottom (magnetogram) plane but contains a highly twisted loop and a shear (current) layer, with a smooth but strong variation of the force-free parameter $\alpha$ in the magnetogram. A standard linear force-free extrapolation of this magnetogram, using the so-called $\alpha_{\text {best }}$ value, is found to fail in reproducing the twisted loop (or flux rope) and the shear layer; it yields a loop pair instead and the shear is not concentrated in a layer. With the nonlinear extrapolation technique, the given equilibrium is readily reconstructed to a high degree of accuracy if the magnetogram is sufficiently resolved. A parametric study quantifies the requirements on the resolution for a successful nonlinear extrapolation. Permitting magnetic reconnection by a controlled use of resistivity improved the extrapolation at a resolution comparable to the smallest structures in the magnetogram.
\end{abstract}

Key words. magnetic fields - magnetohydrodynamics (MHD) - methods: numerical - Sun: corona

\section{Introduction}

The reliable knowledge of the coronal magnetic field is a requirement for progress in the understanding of a wide range of solar phenomena. In particular, the unstable, sometimes catastrophic behaviour of flares and coronal mass ejections is intimately related with the magnetic field properties in the corona (e.g., Priest \& Forbes 2002; Klimchuk 2001; Low 2001). As an example, Török et al. (2004) have demonstrated the possible role of the kink instability of highly twisted flux tubes as an initiation mechanism of flares. It follows that detailed aspects of the coronal field like twist, shear, loop structure, and connectivity have to be known to a high degree of accuracy; it would not be sufficient to investigate the stability of the coronal field based only on integral quantities like its helicity. Unfortunately, due to the extremely low density and high temperature of the corona, measurements of the magnetic field are restricted to lower layers of the solar atmosphere. For this reason, extrapolation techniques, which attempt to reconstruct the coronal field from measured boundary values in the photosphere (or low chromosphere), are the prime tool for quantitative investigations of the coronal magnetic field.

The derivation of the photospheric magnetic field (vector magnetogram) from the Stokes vector of polarized light measured from Earth or from a spacecraft requires the treatment of complex physical models of line formation and radiative transport (see, e.g., Lites 2000). Several sources of uncertainty and even error exist in such measurements. Their treatment is prior to the extrapolation problem as such, and is not further discussed here. Assuming the consistency and correctness of the vector magnetogram - possibly within given errors - the extrapolation problem is that of finding the static, or even forcefree, magnetic field in a three-dimensional domain from given vector values on the photospheric boundary.

Most of the techniques for the extrapolation of magnetic fields are restricted to the force-free case. In the force-free approximation, finite $\beta$ effects that appear to exist in the photosphere and chromosphere up to altitudes of a few hundred kilometers (Metcalf et al. 1995; Moon et al. 2002) are neglected ( $\beta$ is the ratio of thermal to magnetic pressure). The magnetic field satisfies

$\boldsymbol{\nabla} \times \boldsymbol{B}=\alpha \boldsymbol{B}, \quad \boldsymbol{\nabla} \cdot \boldsymbol{B}=0$

where $\alpha(\boldsymbol{x})$ is a scalar function of space that is constant along each individual field line (which follows directly from Eqs. (1)) but, in general, has different values on different field lines. Geometrically, $\alpha$ is a measure of the torsion of neighbouring field lines around each other (Seehafer \& Staude 1983); it is also related to the twist and the current helicity density of the field (see, e.g., Török \& Kliem 2003).

If $\alpha$ is constant then Eq. (1) is linear and only the knowledge of one magnetic field component, e.g., the normal component, is needed as boundary condition for the extrapolation. 
The particular case $\alpha=0$ yields a potential field, which does not contain any free energy to power coronal activity. Reconstructing coronal magnetic fields based on the linear force-free approximation (with $\alpha \neq 0$ ) is still the most widely used approach, and was the only possible extrapolation tool until vector magnetograms became available.

Unfortunately, linear methods have several intrinsic limitations, the most severe one of course being that they cannot match most observed magnetograms closely because the observations show $\alpha$ to be generally strongly varying across active regions (see e.g., Régnier et al. 2002). There are also principal problems at large distances from the magnetogram, where the linear (non-potential) field does not decrease sufficiently rapidly (Seehafer 1978) and the conditions $\boldsymbol{J}=\nabla \times \boldsymbol{B}$ and $\boldsymbol{J}=\alpha \boldsymbol{B}$ cannot be fulfilled simultaneously with constant $\alpha$ because they would then imply different scalings of $\boldsymbol{J}(\boldsymbol{x})$ for $|x| \rightarrow \infty$. Therefore, it is necessary to proceed beyond the linear approximation and to solve the nonlinear extrapolation problem (Sakurai 1981; McClymont et al. 1997; Amari et al. 1999).

A detailed review of nonlinear extrapolation methods is beyond the scope of the present paper. Here only a few key aspects of the most recent methods are mentioned, and we refer to McClymont et al. (1997) for a more complete overview. Nonlinear extrapolation methods can be broadly classified into iterative methods, which iterate approximate solutions of Eq. (1), and evolutionary methods, which seek the solution to Eq. (1) in the form of an asymptotic equilibrium of the timedependent, magneto-hydrodynamic (MHD) equations. While iterative methods now have a more solid mathematical foundation, evolutionary methods offer more flexibility and can in principle easily be extended to include additional physics, such as finite- $\beta$ effects, and to allow for uncertainties in boundary conditions. Additionally, the direct integration method (see, e.g., Wu et al. 1985; Amari et al. 1998) attempts to solve the boundary value problem Eq. (1) as an initial value problem in the height coordinate, which leads to an ill-posed mathematical problem. The errors in the extrapolation grow exponentially with the altitude on a length scale that is comparable with the size of the magnetogram, in this way limiting the practical applicability of the direct integration method to low altitudes.

Two examples of iterative methods are the boundary integral (Yan \& Sakurai 2000) and Grad-Rubin (Sakurai 1981) methods. The boundary integral method is conceptually an extension to the nonlinear case of the solution of the linear forcefree field equations. The method exploits Green's theorem by introducing auxiliary functions through which the solution in each point is expressed by integrals. The Grad-Rubin iterative method (Aly 1989; Aly \& Seehafer 1993; Amari et al. 1999) is based on disentangling the mixed hyperbolic-elliptic character of Eqs. (1). For a given magnetic field distribution, $\alpha$ is determined to be constant along field lines, i.e., the hyperbolic part of Eqs. (1) is solved; then for the new current distribution the field is recalculated, which corresponds to the elliptic part of the equations. The procedure is iterated until convergence is obtained.

Evolutionary methods (McClymont \& Mikic 1994; Mikić \& McClymont 1994), on the other hand, evolve an initial approximation of the field, usually a potential one, into a nonlinear force-free field by integrating the MHD equations, often in an approximate form. Boundary conditions are changed in time so as to eventually match the magnetogram, and a (usually artificial) dissipative mechanism is employed to drive the system toward equilibrium. The tacit and by no means obvious - assumption is that the final, nonlinear state is dynamically accessible as asymptotic equilibrium. Roumeliotis (1996) developed an evolutionary method, referred to as "stress-and-relax" method, which can be regarded as a simplification of the evolutionary method of McClymont \& Mikic (1994). The stress-and-relax method in the formulation of Roumeliotis retains of the MHD equations only those terms that are strictly necessary to obtain pure force-free equilibria. Wheatland et al. (2000) derived an optimization approach based on a pseudo-energy integral which is minimized by a divergence-free, force-free field. The variation of the integral with respect to the variation of the magnetic field gives a pseudo-force, which can be used to dynamically drive the system from a potential field toward a force-free field that minimizes the pseudo-energy integral, subject to the required boundary conditions at the photospheric level. The actual evolution equations used in the optimization approach have little to do with the MHD equations, but the final state is indeed a solution to them. This optimization approach was extended by Wiegelmann (2004) to include coronal density structure and more flexible boundary conditions at the side and top boundaries.

In this paper we present a new implementation of the stressand-relax method, and a detailed analysis of a first application to extrapolate a numerically constructed force-free magnetic configuration which contains a highly twisted loop and a layer of strongly sheared field. Since most models of coronal mass ejections and flares require or predict the formation of a twisted flux rope and involve also highly sheared fields (see, e.g., Forbes 2000), it is important to show that the reconstruction of magnetic field configurations containing such localized current-carrying structures is possible. We choose to adopt the stress-and-relax approach by Roumeliotis because it can be generalized straightforwardly to include pressure and density stratification, finite resistivity effects, and can take account of measurement errors. For this method, it has not yet been shown that the system of equations and boundary conditions constitutes a mathematically well posed problem, but this limitation is shared with all other methods as far as we know. The main difference between the original formulation of the stress-andrelax method and our implementation is that we use the magnetic induction field vector $\boldsymbol{B}$ instead of the vector potential, as originally in Roumeliotis (1996). This keeps errors that result from taking the numerical derivative of the (usually noisy) magnetogram at the necessary minimum. The easier and more direct handling of boundary conditions, which stems from using the magnetic field directly, has the obvious drawback that the solenoidal property must be numerically controlled and imposed to an acceptable level of accuracy. Although only pure force-free applications are reported here, the method is presented in a formulation which includes pressure and inertial effects. 
The content of the paper is the following. In Sect. 2 the method is summarized and details about its numerical implementation are given. In Sect. 3, an application to the extrapolation of a pure force-free field is reported. Additionally, the dependence of the quality of the extrapolation on the resolution is quantified and the possibility to exploit a finite resistivity is demonstrated. Conclusions are presented in Sect. 4.

\section{Implementation of the stress-and-relax method}

\subsection{The stress-and-relax method}

The magnetic field is initialized in a numerical box with a potential or linear force-free field which, by construction, reproduces the normal component of the vector magnetogram at the bottom boundary, but not the tangential ones. The tangential components are then changed to approach or fully match the vector magnetogram, which stresses the field at the bottom of the box. By letting the field in the box evolve in time under the new boundary condition, Lorentz forces are injected into the whole domain which drive the system away from the initial equilibrium. Asymptotically the system tries to relax the imposed stresses in the whole domain. A series of stress-and-relax cycles can be applied until the tangential components of the magnetogram are matched to a pre-defined degree. The flows generated by the Lorentz forces have to be dissipated away for the relaxation to reach a static state that is a solution of the MHD equations. A dissipative term, $\boldsymbol{D}(\boldsymbol{v})$, must therefore be included in the momentum balance equation,

$\rho\left(\frac{\partial v}{\partial t}+\boldsymbol{v} \cdot \boldsymbol{\nabla v}\right)+\nabla p=\boldsymbol{J} \times \boldsymbol{B}+\boldsymbol{D}$.

This term must vanish for vanishing velocities, but can otherwise be specified relatively arbitrarily, since intermediate states of the field in the course of the stress-and-relax procedure are considered to possess no physical significance. Both friction and viscosity terms have been used for $\boldsymbol{D}$, see, e.g. Roumeliotis (1996); Amari et al. (1996). If an asymptotic, static equilibrium is reached, then the surviving terms in the momentum balance equation are the Lorentz force and the pressure term. In this formulation the stress-and-relax method includes nonuniform pressure and is conceptually equivalent to the evolutionary technique in McClymont \& Mikic (1994) and McClymont et al. (1997), except possibly for a different implementation of the boundary conditions. If the additional assumption of low plasma $\beta$ is made, then the pressure term is neglected and the field reduces to the force-free case, Eqs. (1). Only applications to force-free fields are considered in the present paper.

An important point is that reconnection might be needed for a successful extrapolation, because the transition from an initial potential field to a final nonlinear force-free one can in general require a change of field line connectivity, i.e., of field topology (see, e.g., Roumeliotis 1996). Similar to the freedom in the choice of the dissipative term in the momentum equation, an artificial form of the resistivity can be included in the induction equation to allow reconnection in a controlled way. As for the dissipative term $\boldsymbol{D}(\boldsymbol{v})$, resistivity should eventually vanish to allow a static state to be reached.

\subsection{Application to force-free fields}

In order to extrapolate pure force-free fields, one can simplify Eq. (2) to obtain the magneto-frictional method (Yang et al. 1986; Klimchuk \& Sturrock 1992). This method uses friction as dissipative term, $\boldsymbol{D}(\boldsymbol{v})=-v \boldsymbol{v}$, where $v$ is a function of space and time, and discards the inertial and pressure gradient terms. In this approximation the momentum balance equation yields the velocity as explicit function of the time-dependent magnetic field

$\boldsymbol{v}=\frac{1}{v} J \times \boldsymbol{B}$,

where only the friction $v$ is left to be fixed. Equation (3) defines the flow which drives the field towards the force-free state. Hence, in this simplified version of the stress-and-relax method, one only needs to advance the induction equation for the magnetic field

$\frac{\partial \boldsymbol{B}}{\partial t}=\boldsymbol{\nabla} \times(\boldsymbol{v} \times \boldsymbol{B})-\boldsymbol{\nabla} \times(\eta \boldsymbol{J}), \quad \boldsymbol{\nabla} \cdot \boldsymbol{B}=0$

with velocity given by Eq. (3) and $\eta(t \rightarrow \infty) \rightarrow 0$. For topologically simple magnetograms, the extrapolation may be successful with $\eta=0$ throughout (but see Sect. 3.3, for a counterexample).

Since $\alpha$ is constant along the field lines of a force-free field, the problem is overdetermined if the magnetogram is not perfectly consistent with the force-free assumption (as is always the case with magnetograms obtained from observations) and the stressing is set to match the tangential field components (i.e., $\alpha$ ) in the whole magnetogram area. The stressing must then be applied in the area of one sign of $B_{z}$ only, or more complex schemes, e.g. introducing weights for the approach to the observed tangential components in the positive and negative $B_{z}$ areas, must be devised.

\subsection{Initial condition}

The natural choice for the initial state is a potential field. Depending on how the missing information about the magnetic field outside the magnetogram area is handled in the boundary conditions, slightly different potential field solutions are obtained (e.g., Seehafer 1982). If the nonlinear force-free extrapolation problem has a unique solution and if the implementation of the relaxation step permits changes of the field at the lateral and upper boundaries, as in our implementation (Sect. 2.4), then the relaxation will drive the field close to that solution for any (reasonable) choice of the initial condition. A linear force-free extrapolation can then be chosen as well, which can be advantageous for the relaxation if one sign of $\alpha$ dominates in the magnetogram. If the field at the boundaries is not free to change during the relaxation, differences in the nonlinear solution are to be expected, especially if flux concentrations exist close to a magnetogram boundary.

In this paper we will use a magnetogram that contains both signs of $\alpha$ in comparable fractions so that an initial potential field is appropriate. To compute this field we use the expressions derived by Seehafer (1978) for the linear force-free field, setting $\alpha=0$. This method provides the divergence-free field 
in a semi-infinite column of rectangular cross section, which has the normal component of the magnetic field as bottom boundary condition. This linear field vanishes at infinity and it has no vertical component at the side boundaries. Different from other linear methods (see, e.g., Sakurai 1989), Seehafer's method does not require flux balance in the magnetogram, a property which is seldom verified in real measurements. On the other hand, it does require that the normal component of the magnetic field at the bottom vanishes in the approach to the magnetogram boundary. In our implementation, this requirement is fulfilled by simply adding a layer of grid points around the magnetogram where the normal component of the magnetic field vanishes.

Any magnetogram obtained from observations generally requires some processing before it can be used as initial and boundary condition for the extrapolation. This includes the 180 degree ambiguity of the transverse component of the measured field, the transformation to heliospheric coordinates of magnetograms measured far from the centre of the disk, and the strong inhomogeneity of the relative error in the measured transverse field components across the magnetogram, which causes inconsistencies of $\alpha(x, y)$ (see, e.g., McClymont et al. 1997 for a detailed discussion of possible sources of error in the magnetogram which can prevent a correct extrapolation). The nonlinear force-free equilibrium used as a test in this paper was obtained numerically, and is therefore not affected by any of these errors.

\subsection{Boundary conditions}

The three components of the magnetic field vector are specified on two layers of ghost cells at all six boundaries (the numerical grid is composed of the mesh, where the equations are advanced, and two layers of ghost cells on each side, where the boundary conditions are prescribed). Let us describe the inner ghost layer first. On the photospheric boundary the general stress-and-relax strategy prescribes how to impose the magnetic field. The normal component of the initial field is identical to that of the magnetogram. The tangential component must be modified in order to match the magnetogram. This matching between the magnetic field at the photospheric boundary and the magnetogram can be done incrementally in a series of alternating stress and relax steps, but we present here results of a single-step stress version where the magnetogram is directly overwritten onto the photospheric boundary before the system is time-advanced (relaxed). The three components of the magnetic field vector on the photospheric boundary are thus held fixed on the inner bottom ghost layer. This single-step instead of a multi-step stress strategy turned out to minimize the computational effort in the applications presented below. The possibility of a single-step stress phase is allowed by our control on the magnitude of the velocity to conform to the Courant criterion (as described in Sect. 2.5), which essentially precludes numerical instabilities due to large gradients at the bottom of the mesh. No significant differences in the relaxed fields were found between a single-step and a multi-step stress-and-relax strategy applied to the extrapolation presented in this paper.
On the side and top boundaries, the tangential components of the magnetic field are linearly extrapolated onto the ghost layers. The normal component is fixed at each iteration by requiring that the solenoidal property is ensured at the outer mesh layers. Note that these boundary conditions allow for field lines crossing the side and top boundaries.

A second layer of ghost points is employed, since in general the induction equation includes diffusive terms which contain second derivatives of the magnetic field. Here, on all six boundaries, the magnetic field is prescribed in the same way as on the first layer of ghost points at the side and top boundaries. The added layer of grid points around the magnetogram, where $B_{z}=0$, lies in the second layer of ghost points at the side boundaries.

In order to inject information on the magnetic field from the boundary into the extrapolation domain we extend the velocity prescription Eq. (3) to the photospheric layer. At the top and lateral boundaries the velocity is set to zero. Additionally the velocity field is windowed in the outer part of the mesh by a parabolic decrease toward all the boundaries except $z=0$ in typically three to five grid points. The detailed analysis of the extrapolated equilibria is performed within this windowed domain. The effect of this windowing is to allow a slower modification of the initial field close to the non-photospheric boundaries. It permits extrapolating magnetograms that possess substantial flux concentrations close to a boundary. The drawback of the presence of the window is that in practice it reduces the effective extrapolation domain. If necessary, the magnetogram can be embedded in a larger (e.g., full-disk) magnetogram, which is usually available, see, e.g., Jiao et al. (1997).

In this paper we apply our technique to a numerically constructed force-free equilibrium that is known on the whole grid. Hence, the values of the magnetic field at the side and top boundaries could be used as boundary conditions for the extrapolation. We purposely avoided using this information because it is not available for real magnetograms. Similarly, lateral boundaries are placed relatively close to the structure to be reproduced, as is often the case in applications to measurements. In this sense, the extrapolations reported below were obtained in conditions which are similar to those of actual measurements of the magnetic field in solar active regions.

\subsection{Numerical implementation}

We set forth to implement various stress-and-relax strategies in an available software package for solving (sub)systems of the MHD model. The Versatile Advection Code (Tóth 1996, hereafter VAC) offers this flexibility, and is a finite volume code where sets of evolutionary partial differential equations can be solved in different geometries and dimensions. Several spatial discretizations are available, starting from simple differencing schemes to shock-capturing Flux Corrected Transport (Boris \& Book 1973) and Total Variation Diminishing schemes. Time integration can be explicit, implicit or semi-implicit (Tóth et al. 1998; Keppens et al. 1999). Most recently, its AMRVAC version (Keppens et al. 2003) allows automated grid refinement. 
A Cartesian mesh is adopted for magnetograms of active regions or similar test configurations. For the purpose of extrapolating force-free (rapidly but smoothly varying) magnetic fields by time advancing Eq. (4), the spatial discretization can be a pure second order central differencing combined with a time stepping in a single-step forward Euler manner. As we are interested only in achieving a static equilibrium solution, temporal accuracy is of no concern here. Note that this scheme is unconditionally unstable for any normal advection problem, but as described below, will be tailored for stability on this problem. Note that the more sophisticated discretizations available in VAC which we tried thus far did not lead to significantly better results on the test problem of Sect. 3. The reason is that, e.g., the Total Variation Diminishing Lax-Friedrichs method (Yee 1989) can be thought of as a central difference discretization with added physically motivated ("upwinded") terms which introduce intrinsic numerical dissipation. For normal advection problems, these are necessary for stability. They involve nonlinear limiting operations in the linear reconstruction procedures to obtain cell edge values used in flux expressions. This reconstruction achieves second-order spatial accuracy in resolved smoothly varying regions. As we discuss in Sect. 3, the nonlinear force-free equilibrium considered in this paper has an intrinsic length scale at or even below the resolutions we will employ for the extrapolation. In particular at the lower boundary, where fields will need to expand rapidly and form steep gradients, the numerical dissipation in such schemes was found to be so high locally that a satisfactory global relaxation at affordable resolutions was not in reach. form

In practice, the velocity field from Eq. (3) is chosen in the

$\boldsymbol{v}=c_{y} \frac{\Delta x}{\Delta t}\left(\Delta x \frac{\boldsymbol{J} \times \boldsymbol{B}}{|\boldsymbol{B}|^{2}}\right)$,

where $c_{y}(t)$ is a dynamically adjustable constant, and $\Delta x$ and $\Delta t$ are the spatial and temporal step, respectively. The presence of the time step $\Delta t$ in the velocity Eq. (5) avoids oscillations in the final phase of the relaxation, while the factor $|\boldsymbol{B}|^{2}$ is introduced to speed up the relaxation in low field areas (see, e.g., Yang et al. 1986). The remaining terms are fixed by dimensional considerations. Finally, $c_{y}(t)$ is determined at each time step to comply to the Courant criterion, so that

$c_{y} \leq c_{\mathrm{c}} \frac{1}{\max \left(\Delta x|\boldsymbol{J} \times \boldsymbol{B}| /|\boldsymbol{B}|^{2}\right)}$,

where $c_{\mathrm{c}} \leq 1$ is the (constant) Courant parameter. This construction makes it possible to run free of numerical instabilities at nearly the maximum time step set by the stability criterion. The results presented in Sect. 3 are obtained with $c_{\mathrm{c}}=0.5$.

A time and space dependent resistivity can be included. Here we choose a form such that $\eta$ is proportional to the Lorentz force,

$\eta=c_{\eta}|\boldsymbol{J} \times \boldsymbol{B}|, \quad c_{\eta} \equiv \frac{1}{R m} \frac{\max (|\boldsymbol{v}| / \Delta x)}{\max \left(|\boldsymbol{J} \times \boldsymbol{B}| / \Delta x^{2}\right)}$.

The chosen form of $c_{\eta}(t)$ guarantees that the magnetic Reynolds number $R m$ is constant during the evolution. As the system proceeds toward equilibrium, the resistive time scale becomes longer and longer, as required for reaching a steady state. Additionally, a spatial windowing is employed so that resistivity falls off approaching all but the bottom $(z=0)$ boundaries.

A monitor quantity is needed in the relaxation process in order to decide whether the system has approached a forcefree configuration. The degree of relaxation to a force-free field can be quantified by the weighted average angle between the current and the magnetic field vectors, defined as (Wheatland et al. 2000)

$\sigma_{J}=\frac{\sum_{i} J_{i} \sigma_{i}}{\sum_{i} J_{i}}, \quad \sigma_{i} \equiv \frac{|\boldsymbol{J} \times \boldsymbol{B}|_{i}}{J_{i} B_{i}}$

where the index runs over the grid points within the windowed domain of the velocity. If $\sigma_{J}$ has decreased sufficiently and approaches an asymptotic value, we will consider the system as relaxed. It is also useful to monitor where in the grid the maximum Lorentz force is located and the corresponding local value of $\sigma_{i}$.

Finally, the solenoidal property of the relaxed magnetic field is controlled by a diffusive approach (Marder 1987; Dedner et al. 2002). Any numerically generated divergence is then diffused at the maximal rate consistent with an unchanged stability limit on the employed time step. This results in a term $\left(c_{L} \Delta x^{2} / \Delta t\right) \nabla(\nabla \cdot B)$ added to the right hand side of Eq. (4). The coefficient $c_{L}$ is a constant, set equal to 0.2 in all results reported here, except when stated differently. This source term approach for controlling $\boldsymbol{\nabla} \cdot \boldsymbol{B}$ errors was used and compared to other common source term strategies in several test cases of grid-adaptive MHD computations in Keppens et al. (2003).

\section{Application to an equilibrium containing a twisted loop}

As a necessary intermediate step toward the application of the method described in Sect. 2 to measured magnetograms, a numerically constructed nonlinear force-free equilibrium containing a twisted loop and a shear layer is considered here as test case. It is taken out of a sequence of equilibria described in Török \& Kliem (2003), where also a full stability analysis with respect to the amount of twist is presented. The equilibria were obtained by applying localized horizontal vortex motions in the bottom plane of a box that initially contained a bipolar potential field (created by an antisymmetric pair of vertical dipoles below the box). Two identical vortices were centred at the maxima of the normal field in the bottom plane, $\max \left(\left|B_{z}(x, y, 0)\right|\right)$, and applied for different periods of time to inject different amounts of field line twist. A viscous MHD relaxation (with $\nabla p=0$ ) was finally performed resulting in numerically forcefree equilibria that possess line symmetry with respect to the $z$ axis placed in the middle between the vortices. The flux in the vicinity of the central field line which connects the vortex centres forms a nearly uniformly twisted "inner" magnetic loop. The loop does not have a sharp surface, rather the twist decreases smoothly with distance from the central field line (as a consequence of using smooth vortex profiles). Here the twist, $\Phi=l B_{\phi}(r) /\left(r B_{\zeta}(r)\right)$, is defined with respect to the central field line of length $l$ (using local cylindrical coordinates $r$, 

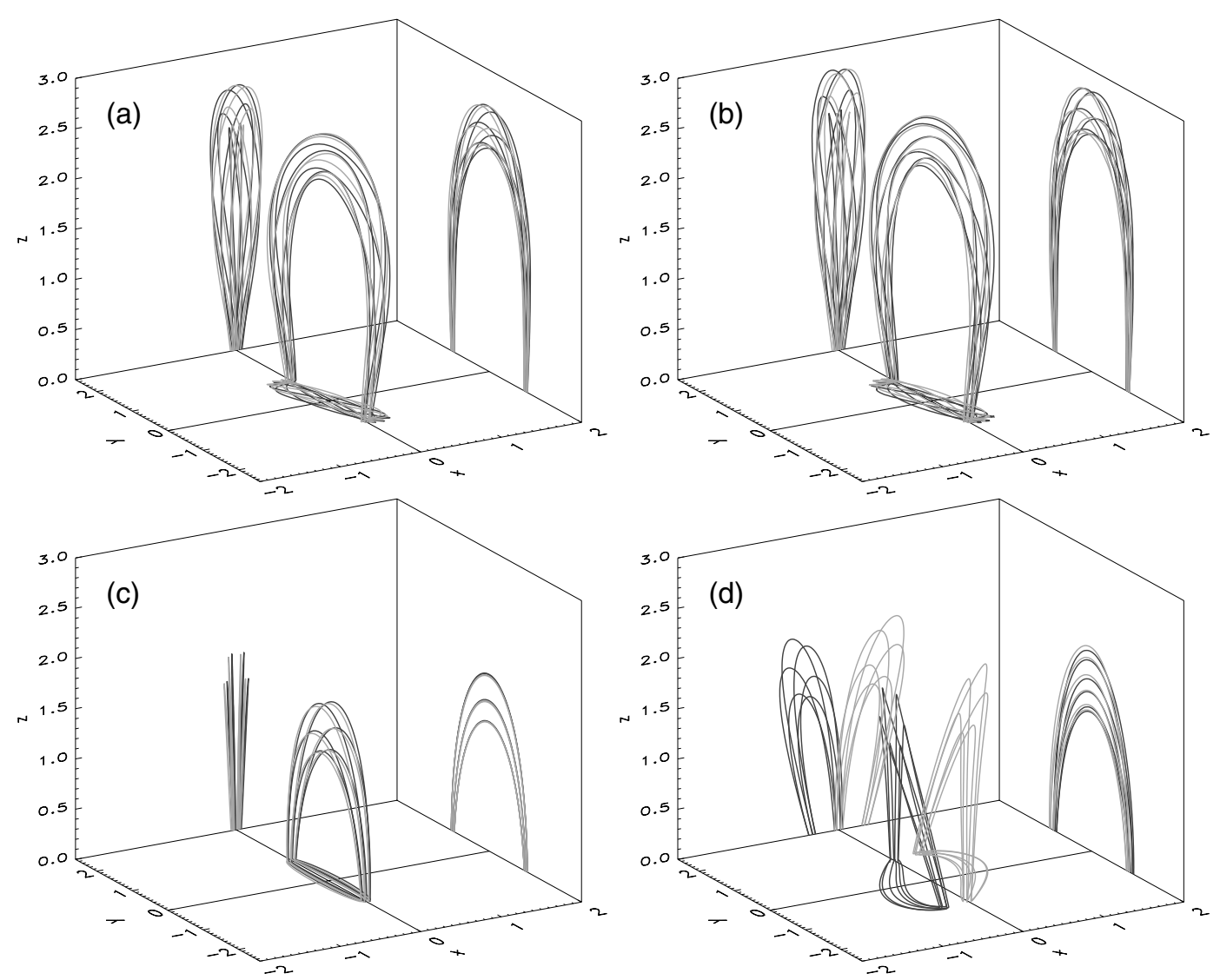

Fig. 1. a) Selected field lines starting at two circles of radius 0.05 centered at the location of maximum twist $(x=0, y= \pm 1.03)$ and their orthogonal projections, for the resolution $2 \Delta$ of the TK equilibrium $(\Delta=0.04)$. b) Nonlinear force-free field extrapolation of the TK equilibrium, resolution $2 \Delta$. c) Potential field constructed from the magnetogram of the TK equilibrium. d) Linear force-free field extrapolation of the TK equilibrium for $\alpha=\alpha_{\text {best }}=-0.51$.

$\phi$, and $\zeta$; see details in Török \& Kliem 2003). By construction, the flux that surrounds the loop carries a diffuse return current of magnitude equal to the current in the loop so that $\alpha$ smoothly changes sign between the inner and outer (also loop-shaped) parts of the current-carrying flux. In addition to the twisted loop, a vertical layer of enhanced current density is formed in the centre of the system, where the vortices shear the field.

Here we consider a single case of a stable configuration (referred to as TK equilibrium in the following) with a (lefthanded) twist in the loop of about $2 \pi$, which amounts to about $80 \%$ of the instability threshold. The magnetic field is normalized such that $\max \left(B_{z}(x, y, 0)\right)=1$. The selected equilibrium is very suitable as a test case for nonlinear extrapolation methods because it possesses high, non-uniform twist and shear and, at the same time, it is a relatively simple magnetic configuration in the sense that the potential bipolar field, which is used as initial field in the extrapolation, and the nonlinear force-free equilibrium are topologically equivalent. Moreover, the field decreases rapidly approaching the side and top boundaries, with the ratio of the maximum value of the magnetic field at the boundaries of the magnetogram to its absolute maximum being about $5 \%$.

Figure 1a shows selected field lines starting at circles of radius 0.05 at the footpoints of the central field line, together with their orthogonal projections. These field lines show the most strongly twisted inner part of the loop. All subsequent field line plots of the twisted structure will use the same start points for the field line integration, and we will use the term "flux tube" in referring to the flux that is enclosed by the surface defined by these field lines. The distribution of $\alpha$ in the bottom plane is displayed in Fig. 2; the strong but smooth variation as well as the sign reversal are apparent.

For the extrapolations that follow, the vector magnetogram is given by the values of the magnetic field at the $z=0$ layer of the TK equilibrium. No further information is used in the reconstruction of this field. Figure 3 shows the contour plot of $B_{z}$ at the magnetogram level. The initial potential field is generated using this $B_{z}(x, y)$ as described in Sect. 2.3, and the corresponding flux tubes, which coincide, are shown in Fig. 1c.

The original data have a uniform resolution of $\Delta=0.04$ in all the directions. A characteristic length scale in the magnetogram can be estimated as $L_{B}=\min \left(\left|J_{z}\right| /\left|\partial_{y} J_{z}\right|\right)$ taken in the plane $z=0$. To compute $L_{B}$ an eleven-point polynomial interpolation of $J_{z}$ in $y$ is taken at the point where $\left|\partial_{y} J_{z}(x, y, 0)\right|$ is maximum. This maximum is located on the flanks of the negative $\alpha$ peaks in Fig. 2. First, second, and third order polynomial interpolation results in the same value $L_{B}=0.1$.

In addition to the original TK equilibrium at a resolution of $\Delta$, the extrapolations of the TK magnetogram at reduced 


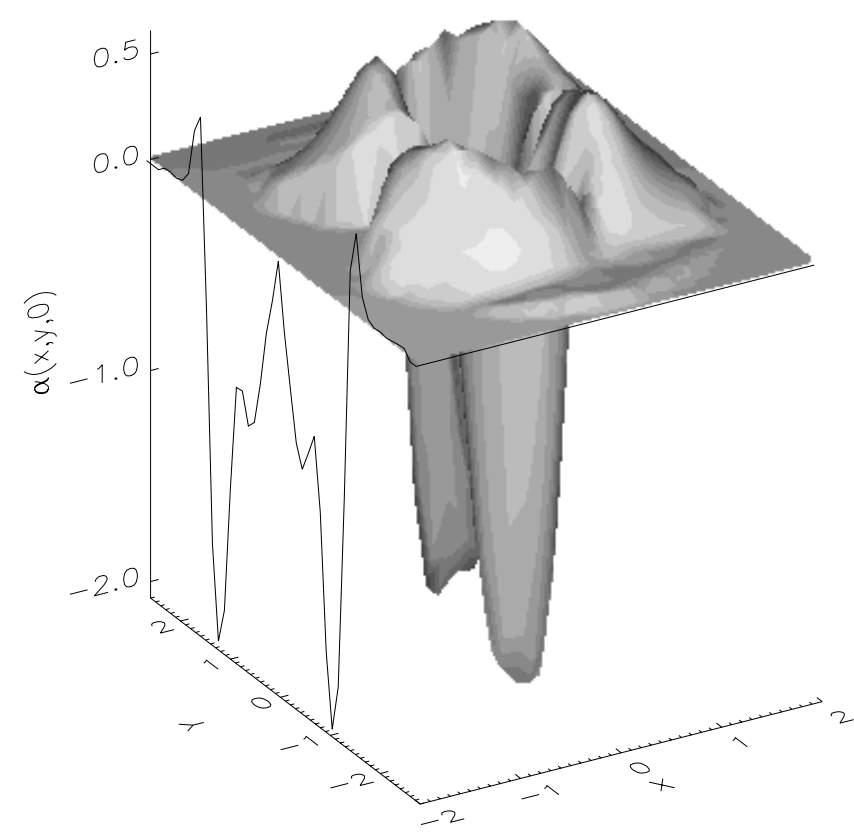

Fig. 2. Surface plot of $\alpha(x, y, z=0)$ for the TK equilibrium, and in thick lines its sections along the axes. In order to avoid the effects of the neutral line, $B_{z}(x, y, z=0)=0$, only points with $\left|B_{z}\right|>0.1 \max \left|B_{z}\right|$ are included.

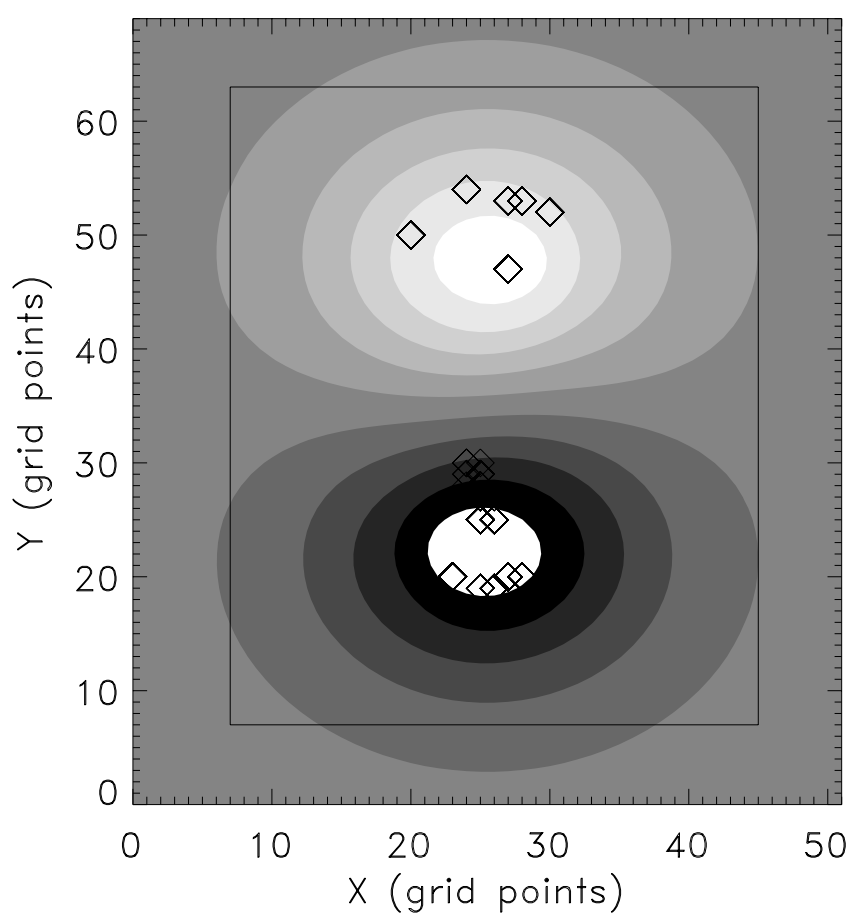

Fig. 3. Contour plot of $B_{z}$ at magnetogram level for the TK equilibrium. The frame shows the sub-domain where the control parameter $\sigma_{J}$ is computed (see text). Diamonds mark the location of maximum Lorentz force at different times during the relaxation process (resolution $2 \Delta$ ) projected on the magnetogram plane.

resolutions of $2 \Delta, 3 \Delta$, and $4 \Delta$ are presented. The magnetograms for the three reduced resolutions are obtained by selecting the field values from the original data at multiple grid spacings. The comparison of the extrapolated fields at different resolutions permits investigating the influence of the resolution on the quality of the extrapolation. This is valuable information because the field on the surface of the Sun always possesses small-scale flux concentrations that cannot be resolved by present-day instruments (see, e.g., Lites 2000). With this in mind, we consider also resolutions ( $3 \Delta$ and $4 \Delta$ ) that exceed the length scale $L_{B}$.

Because of the topological equivalence of the twisted and the initial potential field, we start with extrapolations that use the ideal form of the induction equation ( $\eta=0$ in Eqs. (4)). However, the inclusion of resistivity will be shown to be advantageous in one of the low resolution cases.

Since the magnetogram is consistent with the force-free assumption, the stressing is applied to the whole magnetogram area for faster convergence of the relaxation.

\subsection{Reference case}

The case of resolution equal to $2 \Delta$ is analysed in detail here as an example for all the cases, as it offers good extrapolation results in a reasonably short computational time. The ratio between grid spacing and magnetic field length scale is $2 \Delta / L_{B}=$ 0.8 . The domain is $[-2.04,2.04] \times[-2.8,2.72] \times[-0.08,6$. $]$ on a grid of $(52 \times 70 \times 77)$ nodes.

The flux tube of the extrapolated field is presented in Fig. 1b. The comparison with the flux tube of the TK field in Fig. 1a shows clearly that the extrapolation is successful. The global structure of the field lines is accurately reproduced, including the symmetry with respect to the $z$ axis. This result holds for larger radii of the flux tube $(r=0.05-0.5)$ as well, probing also areas of low values of the magnetic field, within the windowed domain. Figure 4 shows selected field lines through the current layer between the flux tube legs. The field lines for the extrapolated equilibrium (Fig. 4b) are indistinguishable from the field lines of the TK equilibrium (Fig. 4a). The structure of the current layer itself is well reproduced too, as is shown in the isoline plots on the same figure. The ripples visible in the isolines of $|\boldsymbol{J}|$ in Fig. $4 \mathrm{~b}$ are of numerical origin; this is discussed in more detail in Sect. 3.2.

The apex elevation of the central field line eventually approaches a constant level, only $\simeq 7 \%$ off the value 2.47 of the TK equilibrium, as shown in Fig. 5. Note that the field is rapidly decreasing with altitude: at the apex of the central field line it is just $6 \%$ of the value at the footpoints. The extrapolated field reproduces the TK equilibrium nonetheless. In order to check if the twist is correctly obtained, the central field line and a twisting one are plotted in Fig. 6, for the TK and the extrapolated equilibria. This confirms that the extrapolation is remarkably successful in reproducing the high twist of the field.

This extrapolation indeed attains an acceptable relaxation level, as quantified by the evolution of $\sigma_{J}$ and $\sigma_{i}$ in Fig. 7. At this resolution, the average angle between $\boldsymbol{B}$ and $\boldsymbol{J}$, as defined in Eq. (8), drops to 0.015 (0.9 degrees) in the relaxed state. Note that the angle at the location of maximum Lorentz force is just about twice this average. Similarly, the Lorentz force in the final extrapolated solution is about two orders of magnitude smaller than that associated with the central difference discretization errors present in the initial, analytically potential 

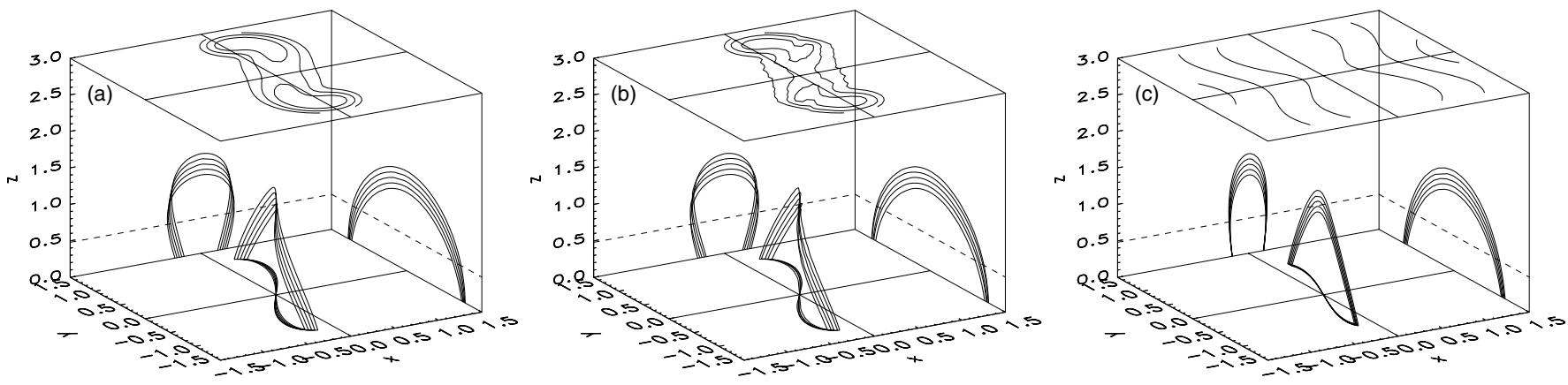

Fig. 4. Field lines crossing the $z$ axis around the maximum of $|\boldsymbol{J}(0,0, z)|$ at $z \simeq 1.2$, and on top isolines of $|\boldsymbol{J}(x, y, 0.48)|$ (at 50,75, and 100 percent of the peak), at resolution $2 \Delta$, for: a) TK equilibrium. b) the nonlinear force-free field extrapolation of the TK equilibrium. c) the linear force-free field extrapolation of the TK equilibrium at $\alpha=\alpha_{\text {best }}=-0.51$.

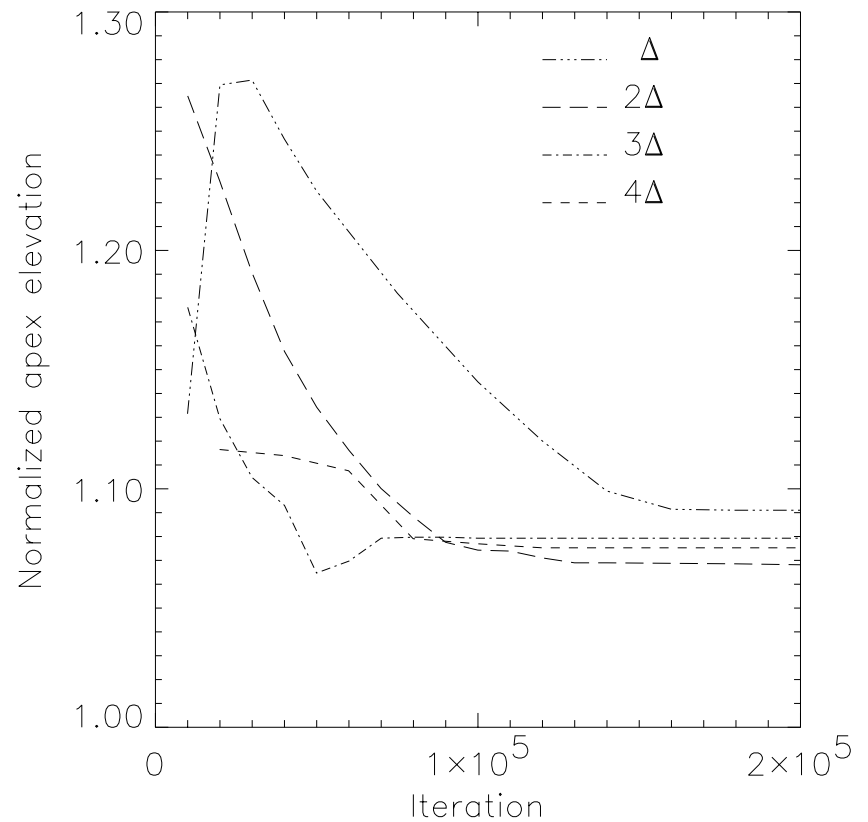

Fig. 5. Evolution of the apex elevation of the central field line of the extrapolated equilibria for different resolutions; the values are normalized to the apex elevation of the TK equilibria at the corresponding resolution. The TK value at resolution $\Delta$ (obtained in Török \& Kliem 2003 with a different numerical relaxation technique) is 2.47 .

field, see Fig. 8. The maximum of the Lorentz force is found at different locations during the evolution. However, all of them are always in the neighbourhood of the field extrema as shown by their projected locations on the magnetogram in Fig. 3. This did require the presence of a window on the velocity (Sect. 2.4), since in the absence of windowing the maximum Lorentz force was invariably found at the side boundaries, introducing errors. The windowing successfully controls this unphysical effect of the boundaries. In the $z$ direction, the maximum of the Lorentz force is almost always found at the second mesh layer $(z=2 \times 2 \Delta)$.

The evolution of the maximum of $|\boldsymbol{\nabla} \cdot \boldsymbol{B}|$ during the extrapolation is also quantified in Fig. 8. This maximum eventually oscillates around a value that is about two orders of magnitude smaller than its initial numerical value of the potential field $\left(4 \times 10^{-2}\right)$. In Fig. 8 the residual of the magnetic field, defined as the root mean square over the mesh and over the three components of the relative variation of the magnetic field between consecutive steps, is also plotted. It is a measure of the field variation, which indeed shows an evolution toward steady state for a large part of the run. Subsequent oscillations are in phase with the more erratic errors of $\max (|\boldsymbol{\nabla} \cdot \boldsymbol{B}|)$. Note that in the total absence of any diffusive $\boldsymbol{\nabla} \cdot \boldsymbol{B}$ treatment this residual settles one order of magnitude below the case shown in the figure, at the totally unacceptable cost of having a magnetic field divergence higher by almost two orders of magnitude.

It is instructive to compare the nonlinear extrapolation result with a linear one obtained using Seehafer's method. It must be recalled that an intrinsic limitation to Seehafer's method comes from the maximum value of $\alpha$ allowed (e.g., Seehafer 1978). The maximum $\alpha$ for a given magnetogram is given by the inverse of the longest half-wavelength fitting into it, so that the linear force-free extrapolation of larger magnetograms is limited to fields with lower $\alpha$. For the case examined, the maximum value of $|\alpha|$ is 0.95 , much smaller than the peak value $\simeq 2$ of the original solution (see Fig. 2). Already for this reason alone, the linear field cannot reproduce the high twist of the original equilibrium. Moreover, both positive and negative values of $\alpha$ of comparable magnitude are present in the magnetogram, hence it is expected that a linear force-free extrapolation cannot reproduce the whole structure of the TK equilibrium. In the literature, a best fitting $\alpha$ is often considered which, in a root mean square sense, reproduces the transverse components on the magnetogram best (Pevtsov et al. 1995). Assuming that this value is much smaller than the maximum value of $|\alpha|$ allowed, the best fitting $\alpha=\alpha_{\text {best }}$ can be expressed explicitly in terms of the magnetogram values. In Fig. 1d the linear extrapolation for the best fitting, constant $\alpha=\alpha_{\text {best }}=-0.51$ is shown. The linear result is misleading concerning the real structure of the field. Its main fault is that this field actually contains two flux tubes instead of one. Moreover, the current layer between the flux tube legs which is present in the TK equilibrium (Fig. 4a), is practically absent in the linear force-free equilibrium (Fig. 4c).

The comparison between linear and nonlinear extrapolations confirms that the latter is required for the reconstruction of the given highly and nonuniformly twisted and sheared 

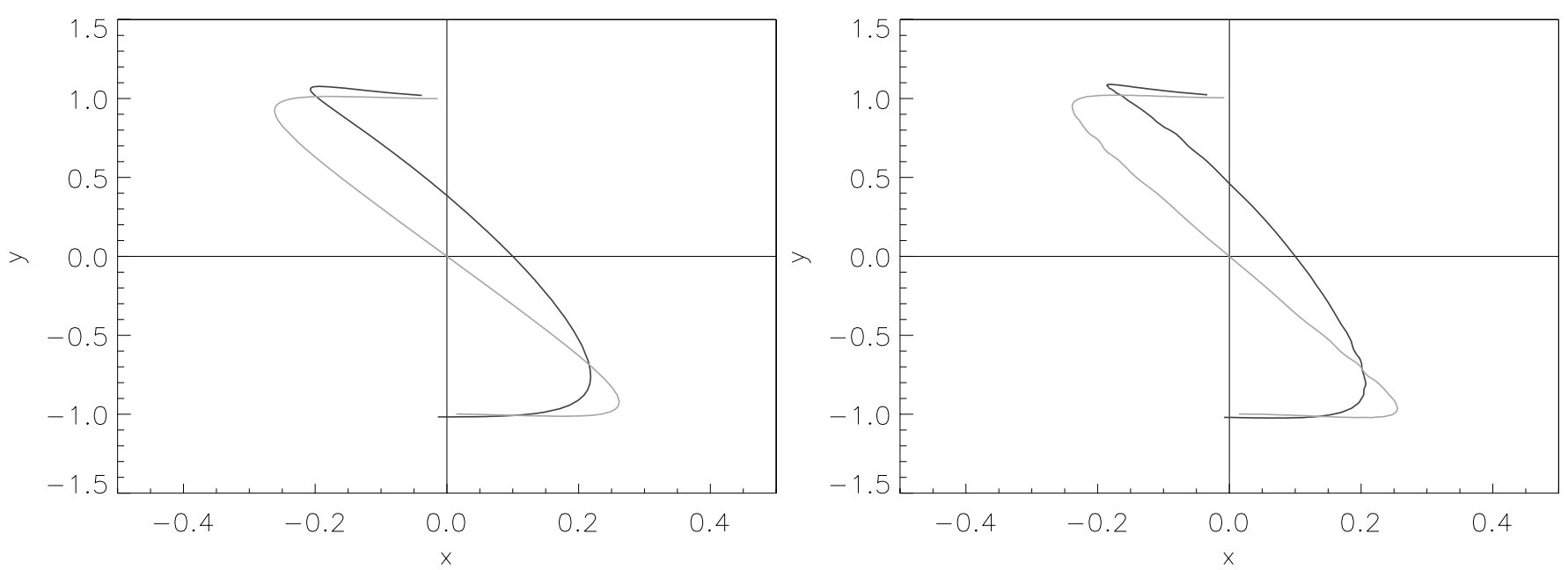

Fig. 6. Projection of the central field line (grey) and a twisting field line (black) for the TK (left) and extrapolated (right) equilibria at resolution $2 \Delta$.

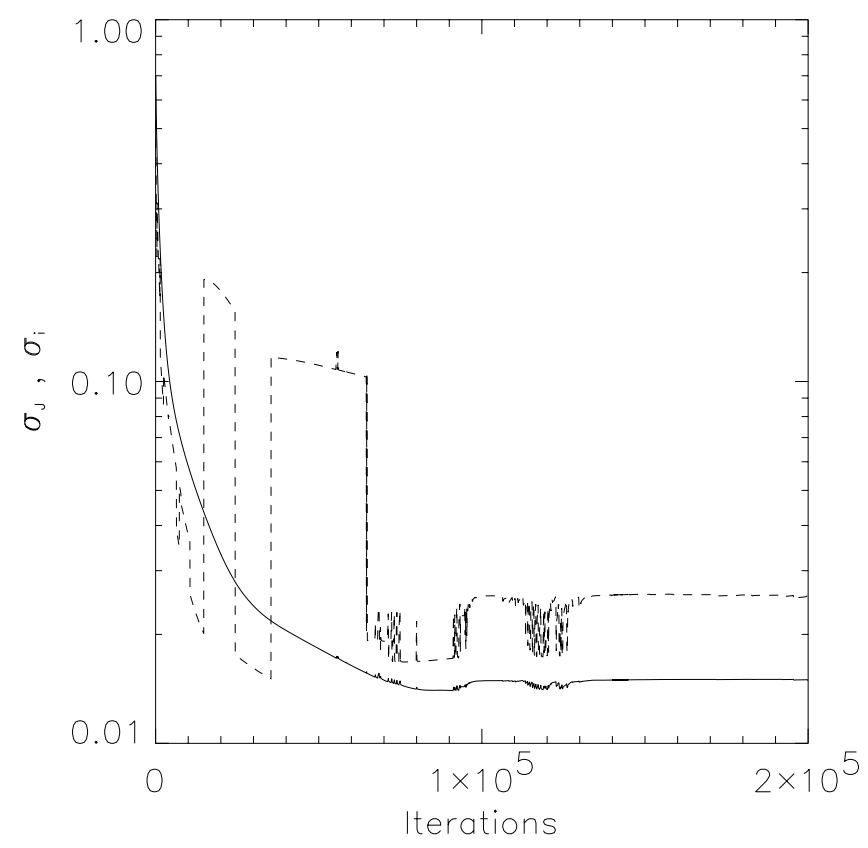

Fig. 7. Evolution of $\sigma_{J}$ (solid line) and $\sigma_{i}$ at the location of maximum Lorentz force (dashes), both as defined in Eq. (8), for resolution $2 \Delta$ in extrapolating for the TK equilibrium.

force-free field, and we have shown that our implementation of the stress-and-relax method in VAC succeeds in this task.

\subsection{Dependence on resolution}

To investigate resolution effects in extrapolation procedures, we now consider extrapolations at grid spacings $\Delta$ to $4 \Delta$. The extrapolation of the TK equilibrium at the original resolution of $\Delta$ is performed using a grid of $(102 \times 140 \times 105)$ nodes for the discretization of the domain $[-2.04,2.0] \times[-2.8,2.76] \times$ $[-0.04,4.12]$. The flux concentrations in the magnetogram are resolved here, $\Delta / L_{B}=0.4$, and one would intuitively say that this is the only case where they are sufficiently resolved. The extrapolation needs a relatively long computational time with

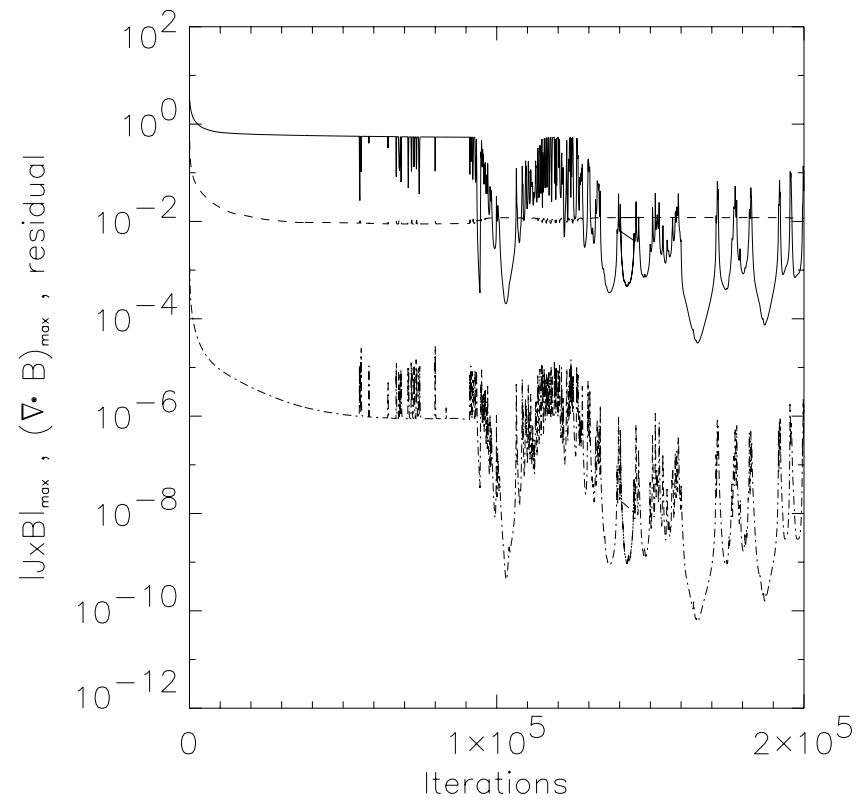

Fig. 8. Evolution of $\max (|\boldsymbol{\nabla} \cdot \boldsymbol{B}|)$ (solid line) and of $\max (|\boldsymbol{J} \times \boldsymbol{B}|)$ (dashes), both normalized to their values in the initial potential field, and of the residual of $\boldsymbol{B}^{2}$ (dot-dashes) for the resolution $2 \Delta$ in extrapolating for the TK equilibrium.

this large grid. In comparison with the reference case, about twice the number of integration steps is required to relax the field and approach the asymptotic state (Figs. 5 and 9). The result is nearly identical: the obtained apex heights differ by only $2 \%$ (Fig. 5) and the field line plot (not shown) is practically indistinguishable from Fig. $1 \mathrm{~b}$.

The quality of the extrapolation degrades for coarser resolutions. The case of resolution $3 \Delta$ is extrapolated using a grid of $(34 \times 46 \times 50)$ nodes on the domain $[-2.04,1.92] \times$ $[-2.8,2.6] \times[-0.12,5.76]$ (somewhat larger box heights raise the computational effort only moderately with the smaller grids of the lower-resolution cases and are chosen to minimize possible boundary effects). Here the grid spacing exceeds the length scale of the loop in the magnetogram, $3 \Delta / L_{B}=1.2$. 


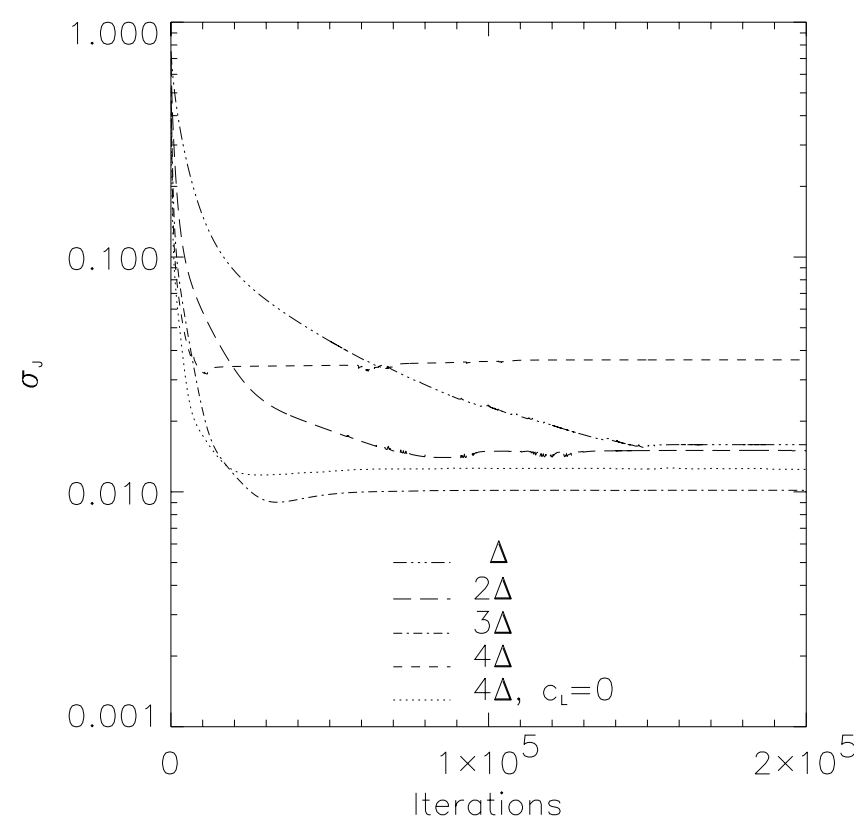

Fig. 9. Evolution of $\sigma_{J}$ for different resolutions and $c_{L}$ values.

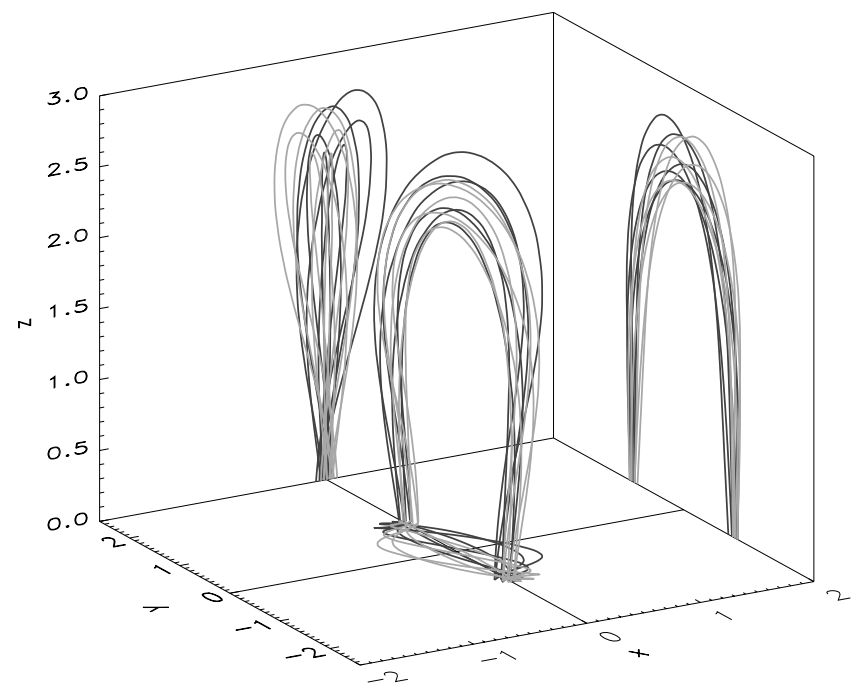

Fig. 10. As Fig. $1 \mathrm{~b}$ for the nonlinear force-free extrapolation of the TK equilibrium at resolution $3 \Delta$.

The extrapolated field does not correctly reproduce the flux tube, which is obvious from its splitting, as Fig. 10 shows. The splitting arises from the non-symmetric location, with respect to $y=0$, of the subset of grid points for this case of an odd multiple of the original grid spacing $\Delta$, which also implies a different sampling of the two footpoint areas. Consequently, the two flux tubes calculated from the two footpoints of the loop in the TK equilibrium do not coincide. Of course, the non-symmetric sampling of the TK magnetogram could have been avoided by using a strictly symmetric grid, but the chosen geometry clearly shows the increasing risk of introducing asymmetry by increasingly coarse resolution. Moreover, it offers a case for testing whether magnetic reconnection can permit the merging of the two flux tubes (see Sect. 3.3). Except for the splitting, the reconstructed flux tube pair does not differ much from the flux tube in the reference case (compare the overall shape, the apex height, and the twisting pattern with Fig. 1b).

The case of resolution $4 \Delta$ corresponds to a grid of only $(26 \times 36 \times 64)$ nodes on the domain $[-2.04,1.96] \times[-2.8,2.8] \times$ $[-0.16,9.92]$, with $4 \Delta / L_{B}=1.6$. The comparison of the flux tube in the TK equilibrium at this resolution in Fig. 11a with the extrapolated one in Fig. 11b shows that the extrapolation succeeds surprisingly well, in spite of the insufficient resolution of the flux concentration in the magnetogram and given the fact that the presence of the window on velocity (Sect. 2.4) limits the volume of deep relaxation to a sub-grid that has just $(16 \times 26 \times 59)$ nodes. The normalized apex heights at the four considered resolutions (Fig. 5) scatter by only $\sim 1 \%$ about their mean. (They are all offset from the corresponding apex height in the TK equilibrium by $7-9 \%$. This systematic difference is probably due to the different relaxation techniques used here and in Török \& Kliem 2003.) We suppose that such an encouraging result can only be obtained in a topologically simple configuration like the TK equilibrium. Numerical diffusion does not easily cause magnetic reconnection in this configuration (not even on this coarse grid). Moreover, the outer parts of the current-carrying field are characterized by length scales somewhat larger than $L_{B}$ and are better reproduced on the coarse grid than the vicinity of the central field line, with the result that the central field line has to sit nearly in the right place. It should be noted that the slight asymmetry of the flux tube(s) visible in both Figs. 11a and b does not reflect an asymmetry of the field values but only an asymmetry of the calculated field lines, which arises primarily from interpolating field values between grid points (with an additional very small contribution from roundoff errors in the field line integration routine).

As a numerical aside, the resolution also influences the effect of the diffusive solenoidal treatment on the final extrapolation. This is most obvious for the case of resolution $4 \Delta$. With $c_{L}=0$ (hence, a purely numerical experiment where no $\boldsymbol{\nabla} \cdot \boldsymbol{B}$ control is performed throughout) the relaxation level is quite good, reaching a $\sigma_{J}=0.013$, see Fig. 9, at the expense of the solenoidal property being violated to a maximal value of $\max (|\boldsymbol{\nabla} \cdot \boldsymbol{B}|)=0.2$. On the other hand, with the advocated choice where $c_{L}=0.2$, the relaxation is "degraded" to $\sigma_{J}=0.04$ but has managed to reduce $\max (|\boldsymbol{\nabla} \cdot \boldsymbol{B}|)=10^{-5}$. The differences in $\sigma_{J}$ obtained with or without diffusive solenoidal treatment are even smaller in the higher resolution runs.

Our parametric study of the influence of the resolution shows that a small value of the global relaxation measure $\sigma_{J}$ is not a sufficient criterion for the correctness of the extrapolation and may in certain cases be somewhat misleading. This is most obvious from our configuration at resolution $3 \Delta$, which achieves the lowest $\sigma_{J}$ (Fig. 9) but produces a split flux tube pair as described above. Moreover, as Fig. 9 indicates, there seems to be a limit on $\sigma_{J}$ levels reachable in our current implementation of the extrapolation procedure which cannot be reduced significantly by simply improving the resolution.

The numerical values of the residual Lorentz forces (which must ideally be zero everywhere) are comparable in the various resolutions, see Fig. 12. The figure shows the absolute value of the Lorentz force vs. height along vertical lines starting at the location of maximum Lorentz force, at the iteration $2 \times 10^{5}$ of 

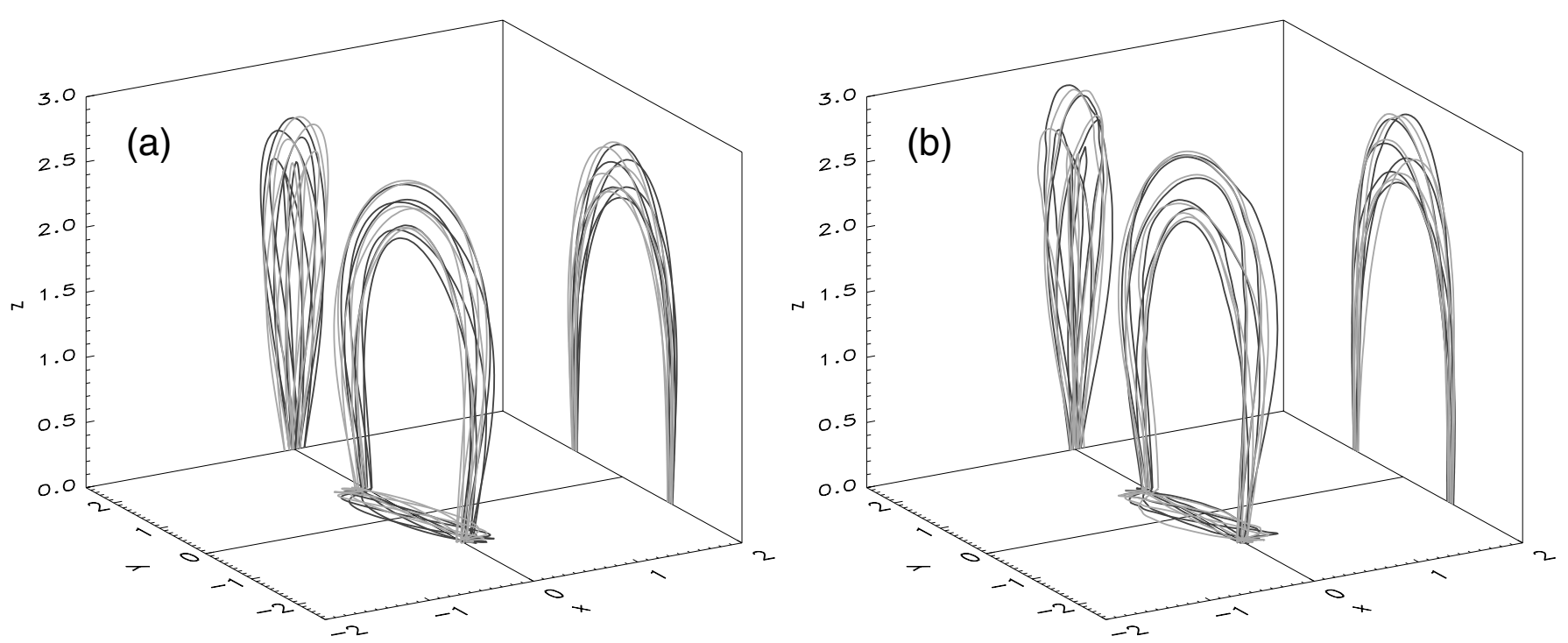

Fig. 11. a) TK equilibrium at resolution $4 \Delta$. b) Nonlinear force-free field extrapolation of the TK equilibrium at resolution $4 \Delta$.

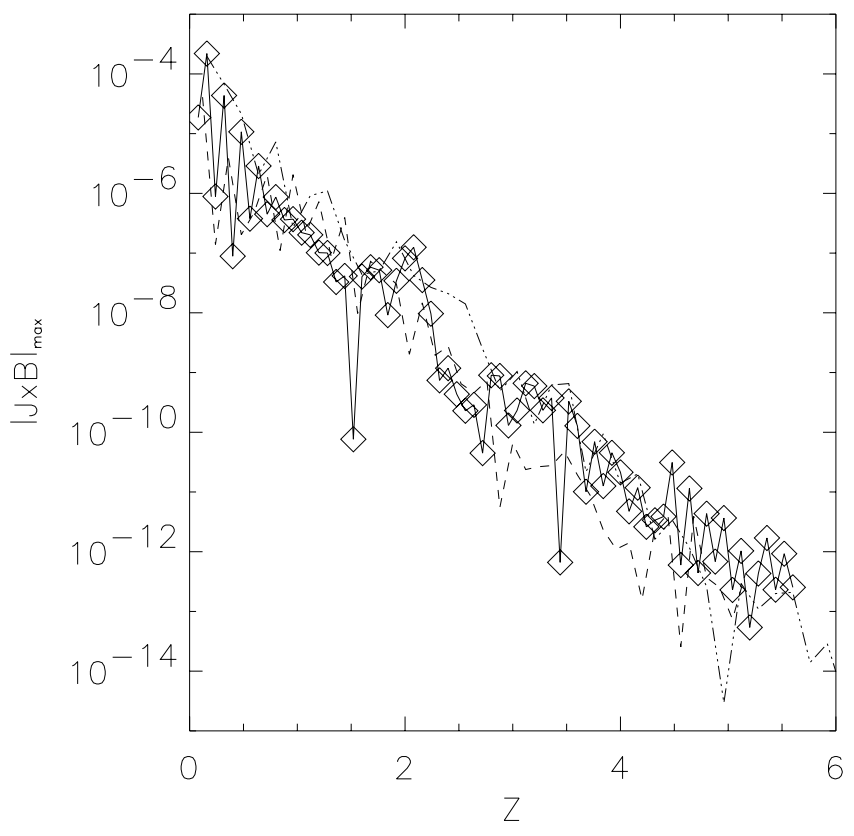

Fig. 12. Variation of the absolute value of the Lorentz force with altitude, along vertical lines starting at the maximum Lorentz force location, at iteration $2 \times 10^{5}$, for the resolution levels $2 \Delta$ (connected diamonds), $3 \Delta$ (dashes), and $4 \Delta$ (dash-dotted).

each run. Not only are the Lorentz forces comparable in magnitude, but they fall off in the same way, roughly exponential, with altitude. There is an oscillation of the discretized Lorentz force with height. This stems from the use of the simple central differencing, leading to an even-odd grid point decoupling, which shows up most clearly in the discretized derivatives of the magnetic field, i.e., in the current density. The effect is strongest close to the photospheric boundary $(z=0)$. This numerical artifact is present in the altitude variation of the Lorentz force and all derived quantities, in particular $\sigma_{i}$. This is likely an intrinsic limitation of the adopted central discretization and

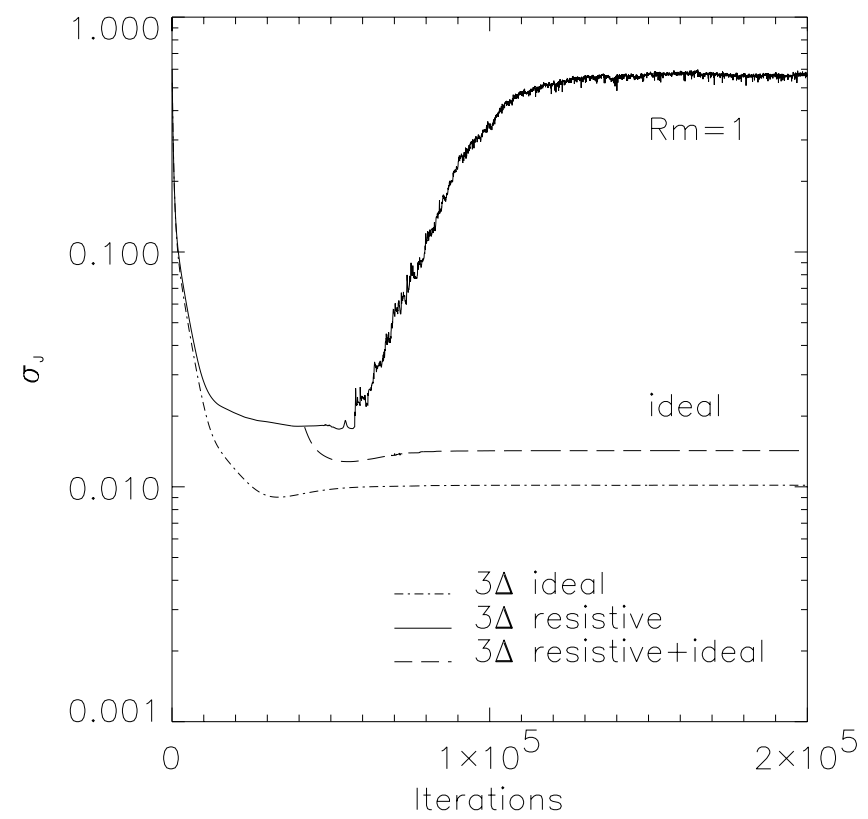

Fig. 13. Evolution of $\sigma_{J}$ for different $R m$ at resolution $3 \Delta$. The solid line shows the late non-force-free evolution for a fully resistive run with $R m=1$. The dashed line shows the mixed resistive-ideal run described in the text.

may explain why the overall relaxation did not improve at resolution $\Delta$, as observed above. Although we made experiments with several possibilities to smooth such oscillations, their effect on the relaxation has so far been found counterproductive.

Only moderate running times were required for the extrapolations reported here: resolution $4 \Delta$ required a few hours on a standard personal computer, while resolution $2 \Delta$ required a few hours of CPU time on a 4-processor vector machine, both up to a total number of $10^{5}$ iterations. 


\subsection{Effect of resistivity}

In the case of resolution $3 \Delta$ a split flux tube pair resulted from using the coarse asymmetric grid. We therefore experimented with the resistive term included to correct this topological defect. When using a constant value of the magnetic Reynolds number $R m=1$ throughout the evolution, it is shown in Fig. 13 that $\sigma_{J}$ reaches a minimum and then grows to large values. In the long run the system is clearly not force-free anymore. However, at the minimum of $\sigma_{J}$, the two flux tubes starting from the symmetric circles actually coincide, as is the case in the TK equilibrium. Continuing with the evolution from this minimum state with $R m=\infty$, the final extrapolation result turns out to be correct: the final field line plot (not shown) is nearly identical to the case of resolution $2 \Delta$ in Fig. $1 \mathrm{~b}$. The difference in the final apex elevation between this hybrid resistiveideal run and the reference case is about 1\% (see Fig. 5). Hence, the initial change in field line connectivity allowed by resistivity manages to recover the original structure of the TK configuration despite the use of an asymmetric grid. In contrast to this encouraging result, it turned out that the inclusion of resistivity was counterproductive at the coarsest resolution of $4 \Delta$, where the flux tubes finally connected to the side boundaries. This simple experiment already indicates that utilizing resistivity has its limits, especially in underresolved complex configurations with multiple polarity reversals in the magnetogram. Further experiments with test configurations will be necessary to develop reliable strategies of resistive relaxation for application to measurements.

\section{Conclusions}

In this paper we have presented a new implementation of the stress-and-relax method for the extrapolation of coronal fields from photospheric boundary data, originally proposed by Roumeliotis (1996). Here this method is formulated in a manner analogous to the evolutionary extrapolation method by McClymont \& Mikic (1994), and its implementation in the numerical package VAC is described. The method is successfully applied to the force-free extrapolation of a test magnetogram taken from a numerical nonlinear force-free equilibrium that contains a highly twisted magnetic loop and a shear layer in a topologically simple bipolar configuration. The twist in the vicinity of the loop axis is approximately $2 \pi$, that is about $80 \%$ of the maximum twist permitting a stable equilibrium of this type. The chosen configuration offers the combined challenge of a strongly nonuniform distribution of $\alpha$ at the photosphere together with strongly helical field line shapes. We have shown that the twisted loop and the shear layer can be reproduced with high accuracy by our nonlinear extrapolation procedure. This is contrary to the failure of the linear force-free field extrapolation, which does not reproduce the qualitative features of the original field entirely: either the loop is not twisted and shear is absent $(\alpha=0)$ or the twisted loop splits and the shear is smeared over the volume ( $\left.\alpha=\alpha_{\text {best }}\right)$.

The influence of the resolution on the extrapolation results is investigated as well. The extrapolation of the test magnetogram is found to be accurate as long as the grid spacing is smaller than (or near to) a characteristic length scale, $L_{B}$, of the field in the magnetogram. In situations of moderate underresolution, as in the cases of grid spacing (1.2-1.6) $L_{B}$ analysed here, the method gives the correct global structure of the field, but quantitative differences start showing up. Artificial resistivity can be used to improve the extrapolation in a range of under-resolution, provided the magnetogram is not too complex.

By implementing various strategies for stress-and-relax methods in VAC, we expect to easily extend our extrapolation efforts. More elaborate boundary treatments (such as multistage stress-and-relax stepping, matching the transverse field of only one polarity of the normal field, matching within errors of measurements), and allowing non-uniform pressure distribution have already been included in our code. Applications to more complex test cases as well as to actual magnetogram data will be reported in future publications.

Uniform grids are employed in the calculations reported here. At high resolutions many points are then used in the discrete representation also of low field volumes, with the drawback of very long relaxation times. Although VAC can discretize equations on nonuniform grids, using this option was not attempted because, first, magnetograms are obtained from uniform scannings and, second, each magnetogram would require a different, nonuniform grid to be used, which might have to be changed during the extrapolation process. Instead, a much more efficient technique to resolve strong gradients in unpredictable locations and to save computational effort in low field volumes is offered by using automated mesh refinement. AMRVAC (Keppens et al. 2003) is intended to provide dynamical grid adjustment more efficiently than nonuniform fixed grids, and will be used in future applications.

Acknowledgements. The data of the TK equilibrium (Fig. 1a) were provided by Tibor Török. The authors thank Tibor Török, Axel Hofmann, Norbert Seehafer, and Jürgen Staude for useful discussions, and the referee, Thomas Neukirch, for constructive comments. This work has been supported in part by the European Community's Human Potential Programme under contract HPRN-CT 2000-00153, PLATON. R.K. acknowledges financial support from NWO.

\section{References}

Aly, J. J. 1989, Sol. Phys., 120, 19

Aly, J. J., \& Seehafer, N. 1993, Sol. Phys., 144, 243

Amari, T., Boulmezaoud, T. Z., \& Maday, Y. 1998, A\&A, 339, 252

Amari, T., Boulmezaoud, T. Z., \& Mikic, Z. 1999, A\&A, 350, 1051

Amari, T., Luciani, J. F., Aly, J. J., \& Tagger, M. 1996, ApJ, 466, L39

Boris, J., \& Book, D. 1973, J. Comput. Phys., 11, 38

Dedner, A., Kemm, F., Kröner, D., et al. 2002, J. Comput. Phys., 175, 645

Forbes, T. G. 2000, J. Geophys. Res., 105, 23153

Jiao, L., McClymont, A. N., \& Mikić, Z. 1997, Sol. Phys., 174, 311

Keppens, R., Nool, M., Tóth, G., \& Goedbloed, J. 2003, Comp. Phys. Comm., 153, 317

Keppens, R., Tóth, G., Botchev, M., \& van der Ploeg, A. 1999, Int. J. Numer. Meth. Fluids, 30, 335 
Klimchuk, J. A. 2001, in Space Weather, ed. P. Song, H. J. Singer, \& G. L. Siscoe, Geophys. Monograph, 125 (AGU: Washington), 143

Klimchuk, J. A., \& Sturrock, P. A. 1992, ApJ, 385, 344

Lites, B. W. 2000, Rev. Geophys., 38, 1

Low, B. C. 2001, J. Geophys. Res., 106, 25141

Marder, B. 1987, J. Comput. Phys., 131, 48

McClymont, A. N., Jiao, L., \& Mikić, Z. 1997, Sol. Phys., 174, 191

McClymont, A. N., \& Mikic, Z. 1994, ApJ, 422, 899

Metcalf, T. R., Jiao, L., McClymont, A. N., Canfield, R. C., \& Uitenbroek, H. 1995, ApJ, 439, 474

Mikić, Z., \& McClymont, A. N. 1994, in Solar Active Region Evolution: Comparing Models with Observations, ASP Conf. Ser. 68, 225

Moon, Y., Choe, G. S., Yun, H. S., Park, Y. D., \& Mickey, D. L. 2002, ApJ, 568, 422

Pevtsov, A. A., Canfield, R. C., \& Metcalf, T. R. 1995, ApJ, 440, L109

Priest, E. R., \& Forbes, T. G. 2002, A\&AR, 10, 313

Régnier, S., Amari, T., \& Kersalé, E. 2002, A\&A, 392, 1119

Roumeliotis, G. 1996, ApJ, 473, 1095
Sakurai, T. 1981, Sol. Phys., 69, 343

Sakurai, T. 1989, Space Sci. Rev., 51, 11

Seehafer, N. 1978, Sol. Phys., 58, 215

Seehafer, N. 1982, Sol. Phys., 81, 69

Seehafer, N., \& Staude, J. 1983, Physica Solariterr., 22, 5

Török, T., \& Kliem, B. 2003, A\&A, 406, 1043

Török, T., Kliem, B., \& Titov, V. S. 2004, A\&A, 413, L27

Tóth, G. 1996, Astrophys. Lett. Comm., 34, 245

Tóth, G., Keppens, R., \& Botchev, M. 1998, A\&A, 332, 1159

Wheatland, M. S., Sturrock, P. A., \& Roumeliotis, G. 2000, ApJ, 540, 1150

Wiegelmann, T. 2004, Sol. Phys., 219, 87

Wu, S., Chang, H., \& Hagyard, M. 1985, in Measurement of Solar Vector Magnetic Fields, NASA Conf. Pub., 2374, ed. Hagyard (Marshall Space Flight Center Workshop), 17

Yan, Y., \& Sakurai, T. 2000, Sol. Phys., 195, 89

Yang, W. H., Sturrock, P. A., \& Antiochos, S. K. 1986, ApJ, 309, 383

Yee, H. 1989, NASA TM-101088 